\title{
Extinction of conditioned inhibition: Effects of different outcome continua
}

\author{
AnJa Lotz and HaRald Lachnit \\ Philipps-Universität Marburg, Marburg, Germany
}

\begin{abstract}
For some decades, failures to find extinction of inhibition through unpaired presentations of the inhibitor were taken as evidence against conceptualizing inhibition as the symmetrical counterpart of excitation. Recently, however, our group has demonstrated successful extinction of inhibition in human causal learning. In two experiments, we replicated and strengthened this finding by using an outcome continuum that could take on negative, neutral, or positive values. In contrast, the use of a dichotomous outcome continuum (either neutral or positive) resulted in the well-known nonoccurrence of extinction. Extinction of inhibition through the pairing of inhibitors with neutral outcomes was assessed by (1) comparing the (presumably) extinguished inhibitor with a second inhibitor that had not been presented with a neutral outcome in the extinction stage, and (2) demonstrating the course of extinction in participants' predictions.
\end{abstract}

Conditioned inhibition can be described as the consequence of learning that after a certain event (the CS), another event (namely, the US) will be less likely than usual to occur in a given situation. According to Rescorla and Wagner's (1972) influential theory of associative learning, conditioned excitation and conditioned inhibition are opposite ends of an underlying continuum on which a cue's associative strength, $V$, can vary. In this view, conditioned inhibition is the symmetrical counterpart of conditioned excitation. As a consequence, there should be no fundamental differences between excitation and inhibition. Especially, it should be possible to extinguish both by the same procedure. The standard procedure for extinguishing a conditioned excitor is to present the excitor without the US. Attempts to extinguish a conditioned inhibitor in the same way, however, have repeatedly failed (see, e.g., Miller \& Schachtman, 1985; Zimmer-Hart \& Rescorla, 1974; for an overview, see Williams, Overmier, \& LoLordo, 1992). These failures led many researchers to reject the idea that inhibition is the symmetrical counterpart of excitation. Instead, threshold accounts of inhibition (e.g., Konorski, 1948; Rescorla, 1979) or additional no-US centers (e.g., Konorski, 1967; Pearce, 1987) were suggested. The most radically different account - the comparator theoryexplains inhibition as a behavioral effect that is based on excitatory associations exclusively (e.g., Miller \& Matzel, 1988). Melchers, Wolff, and Lachnit (2006) recently suggested, however, that the reason for the failure to find extinction of conditioned inhibition by presenting the inhibitor without the US might be that in all empirical attempts so far, a unidirectional US or outcome continuum was used.

\section{Extinction of Inhibition and Surprise}

A central assumption of the Rescorla-Wagner theory is that surprise is a precondition of learning. Surprise occurs as a consequence of a discrepancy between the expected and the actual outcome of a learning trial. The unexpected presence of an outcome should result in an increase of the associative strength, $V$, whereas the unexpected absence of an outcome should result in a decrease of $V$. If a $\mathrm{CS}$ is paired with an outcome repeatedly $\left(\mathrm{CS}^{+}\right)$, its $V$ is expected to increase asymptotically because of a discrepancy between $V$ and the asymptotic $V$ supported by the US $(\lambda)$, resulting in excitatory $V\left(\mathrm{CS}_{+}\right) \cdot{ }^{1}$ If this $\mathrm{CS}_{+}$is later presented without the outcome $\left(\mathrm{CS}_{+}^{0}\right)$, there is again a discrepancy between $V$ and $\lambda$. The organism will be surprised and $V$ will decrease, resulting in a neutral $\mathrm{CS}\left(\mathrm{CS}_{0}\right)$ at asymptote. Thus, repeated presentations of an excitatory $\mathrm{CS}$ without an outcome $\left(\mathrm{CS}_{+}^{0}\right)$ will result in an extinction of conditioned excitation. Conditioned inhibition, on the other hand, can be established by presenting a CS without the outcome in a situation in which the outcome is expected. This may be accomplished by the standard paradigm of conditioned inhibition $\left(\mathrm{A}^{+}, \mathrm{AX}^{0}\right)$, in which the putative inhibitor $\left(\mathrm{X}_{-}\right)$is established by unpaired presentation in compound with a conditioned excitor $\left(\left[\mathrm{A}_{+} \mathrm{X}_{-}\right]^{0}\right)$. With this training, the positive $V$ of the excitor $\mathrm{A}_{+}$is assumed to summate with the negative $V$ of the putative inhibitor $\mathrm{X}_{-}$, resulting in a net $V$ of approximately 0 for the $\left[\mathrm{A}_{+} \mathrm{X}_{-}\right]$compound. This way, the expectation matches the actual outcome. Up to this point, the evidence in favor of the Rescorla-Wagner model is quite straightforward.

The crucial point is that, in the case of the unpaired presentation of the inhibitor $\left(\mathrm{CS}_{-}^{0}\right)$, the prediction of the Rescorla-Wagner model (extinction of inhibition through

A. Lotz, lotza@staff.uni-marburg.de 
unpaired presentations of the inhibitor) conflicts with the general principle that surprise is required for learning: On the one hand, unpaired presentations of the inhibitor $\mathrm{X}$ (Xㅜ) should lead to extinction of inhibition because of the discrepancy of the negative $V$ and $\lambda$. On the other hand, the model assumes that surprise is a precondition for learning. In most cases, however, the outcome empirically cannot take on negative values. The negative $V$ accruing in acquisition has not been the result of experience with a negative outcome, but is a result of presentation in compound with an excitor that overpredicted the outcome. In extinction, then, when the putative inhibitor is not followed by the outcome ( $\left.\mathrm{CS}_{-}\right)$, this experience - that is, the absence of the outcome - cannot serve as a "valid test" from the organism's point of view, because less than nothing is not a possible situation empirically. In other words, extinction of inhibition through unpaired presentation of the inhibitor suffers from a floor effect; hence, the empirical outcome matches the expectation: Nothing is expected - because it is impossible to experience less than nothing - and nothing really happens. As a consequence, the organism does not become surprised in extinction trials; hence, no learning (i.e., no extinction) will occur since there is no need to adjust expectations. If anything, the absence of the outcome confirms the expectation that no such outcome should occur.

\section{Effects of Varying Properties of the Outcome}

To resolve this problem and to enable the experience of surprise in extinction of inhibition, Melchers et al. (2006) introduced a new type of outcome continuum, allowing the outcome to take on negative values. They used a human causal learning scenario in which the level of a hypothetical hormone (US) was influenced by the consumption of several foods (CSs). The continuum was either a (traditional) unidirectional continuum, on which the US was either present (hormone level increased) or absent (hormone level did not change), or a bidirectional continuum consisting of three stages: The hormone level could increase, not change, or decrease. Correspondingly, a food that was followed by an increase was referred to as $\mathrm{CS}^{+}$, a food that was followed by no change was referred to as $\mathrm{CS}^{0}$, and a food that caused a decrease was referred to as $\mathrm{CS}^{-}$. The authors were able to demonstrate successful extinction of inhibition through the presentation of the inhibitor with a neutral outcome (CS $\underline{0})$ in the condition with a bidirectional continuum, but not with the unidirectional continuum. Only in case of extinction with the bidirectional continuum is the neutral outcome unexpected $\left(\mathrm{CS}_{-}^{0}\right)$, since the inhibitor $\left(\mathrm{CS}_{-}\right)$can, in principle, be followed by a negative outcome $\left(\mathrm{CS}_{-}^{-}\right)$. Hence, with a bidirectional outcome continuum, there no longer is a floor effect in case of pairings with a neutral outcome ( $\left.\mathrm{CS}_{-}^{0}\right)$. A valid difference exists empirically between pairings of the CS with a neutral outcome or with a negative outcome, and learning can therefore take place.

In a similar vein, other authors have investigated relations between assumptions about outcome properties and learning. Mitchell and Lovibond (2002), for example, in- vestigated such expectations in blocking experiments. In a blocking procedure, a CS, A, is repeatedly paired with an outcome $\left(\mathrm{A}^{+}\right)$, in the experimental condition. After this training, $\mathrm{A}$ becomes an excitor $\left(\mathrm{A}_{+}\right)$which thereafter is presented in compound with another stimulus, $\mathrm{B}$, and this compound is also paired with that outcome $\left(\left[\mathrm{A}_{+} \mathrm{B}\right]^{+}\right)$. Successful blocking is assumed if, in a subsequent test, the response elicited by $\mathrm{B}$ is weaker than the response elicited by $\mathrm{B}$ in a control condition without prior $\mathrm{A}^{+}$training. Mitchell and Lovibond found that the strength of blocking was related to the assumptions of the participants as to whether or not the outcomes were additive: If participants were told that the presence of two valid predictors of the outcome would lead to the application of a double outcome $\left(\mathrm{AB}^{++}\right)$, blocking was stronger than it was in conditions in which participants were encouraged to assume that outcomes were not additive and could not be larger than a certain (ceiling) value. In the first condition, during the $\left[\mathrm{A}_{+} \mathrm{B}\right]^{+}$trials, participants could rule out that $\mathrm{B}$ had an effect on its own. This assumption was not possible in the latter condition, because of the imposed ceiling. Thus, expectations about the outcome magnitude can influence what is learned about certain cues. In contrast to extinction of inhibition (in which a unidirectional continuum produces a floor effect), in blocking studies, results often are affected by ceiling effects.

In a further causal learning study, Lovibond, Been, Mitchell, Bouton, and Frohardt (2003) examined the effect of pretraining (additive vs. nonadditive) on blocking by training either $\mathrm{I}^{+}, \mathrm{J}^{+}, \mathrm{IJ}^{++}$(additive) or $\mathrm{I}^{+}, \mathrm{J}^{+}, \mathrm{IJ}^{+}$ (nonadditive) prior to the blocking procedure. A single outcome $\left(\mathrm{CS}^{+}\right)$was implemented by specifying that an "allergic reaction" would follow the consumption of a given food; the double outcome $\left(\mathrm{CS}^{++}\right)$was implemented by specifying a "strong allergic reaction." Lovibond et al. found a stronger blocking effect in the additivity condition than in the nonadditivity condition. The results show that manipulations of outcome properties, such as variations of the underlying continuum (with either + or ++ as the end point), are relevant in studies of associative learning.

In the present article, we report two experiments with varying properties of outcome continua investigating extinction of inhibition through pairing of the inhibitor with a neutral outcome. One objection raised against the conclusions of Melchers et al. (2006) could be that it seems hard to make any claims about extinction when one does not have a comparison condition that lacks the extinction treatment. Although they got differences as a result of their primary manipulation that are consistent with a difference in extinction, they did not include controls that unambiguously demonstrated the operation of an extinction process. Therefore, the first experiment was conducted to test whether extinction of inhibition is cue specific. If so, then a second inhibitor established during acquisition - but not paired with a neutral outcome during the extinction stage - could serve as a within-subjects control for extinction. Furthermore, we aimed to demonstrate extinction directly by assessing performance over the course of extinction. 


\section{EXPERIMENT 1}

Experiment 1 was conducted to replicate and expand the findings of Melchers et al. (2006) by introducing two further tests for the extinction treatment. A potential shortcoming of the study of Melchers et al. was the fact that extinction was shown by comparing the putatively extinguished inhibitor between groups. In Experiment 1, we used a second inhibitor that was not paired with a neutral outcome in the extinction stage. This way, the potentially extinguished inhibitor, could directly be tested against the second inhibitor, allowing a within-subjects comparison at the time of testing. In addition, we aimed at inspecting the course of extinction in scoring the responses made by the participants during the course of the procedure. We used the same medical prediction task as did Melchers et al., which is similar to the task used in many human causal learning studies (e.g., Aitken, Larkin, \& Dickinson, 2000; Melchers, Lachnit, \& Shanks, 2004). In this task, participants had to learn which of several foods cause a change in the hormone levels of hypothetical patients. In Group Unidirectional, the hormone level could either increase or remain unchanged, which parallels the conditions from earlier causal learning studies investigating inhibition (e.g., Aitken et al., 2000; Chapman \& Robbins, 1990). In Group Bidirectional, in contrast, the hormone level could increase, remain unchanged, or decrease. Thus, potential expectations based on positive, neutral, or negative values of $V$ are empirically mirrored by the values the outcome could take on.

The experiment consisted of an acquisition stage and an extinction stage, both followed by a rating phase (see Table 1). During acquisition, two cues-X and $\mathrm{Y}-$ were trained in the standard paradigm of conditioned inhibition $\left(\mathrm{A}^{+}, \mathrm{AX}^{0}\right.$ and $\left.\mathrm{B}^{+}, \mathrm{BY}^{0}\right)$, where ${ }^{0}$ stands for a neutral outcome ("level remains unchanged"). This procedure should make $\mathrm{X}$ and $\mathrm{Y}$ inhibitory $\left(\mathrm{X}_{-}, \mathrm{Y}_{-}\right)$and $\mathrm{A}$ and $\mathrm{B}$ excitatory $\left(\mathrm{A}_{+}, \mathrm{B}_{+}\right)$(see, e.g., Karazinow \& Boakes, 2004). A further cue to be used for a later summation test was presented with a positive outcome $\left(\mathrm{T}^{+}\right)$. Furthermore, filler trials ensured that participants experienced pairings of a single cue with a neutral outcome as well as pairings of a compound with a positive outcome $\left(\mathrm{C}^{0}, \mathrm{DE}^{+}\right)$. Last, but not least, an additional filler cue, F, was used to emphasize the unidirectional or bidirectional nature of the outcome, respectively. In Group Unidirectional, the hormone level remained unchanged after presentations of $\mathrm{F}\left(\mathrm{F}^{0}\right)$, whereas in Group Bidirectional, the hormone level decreased $\left(\mathrm{F}^{-}\right)$. In the test, inhibition was assessed with direct ratings of the putative inhibitors $\mathrm{X}$ and $\mathrm{Y}$, and with a summation test by presenting $\mathrm{X}$ and $\mathrm{Y}$ in compound with the excitatory cue $\mathrm{T}$.

During the extinction stage, $\mathrm{X}^{0}$ trials were presented instead of $\mathrm{AX}^{0}$ trials. Training of the other cues proceeded as before, with the exception that neither $\mathrm{BY}^{0}$ nor $\mathrm{DE}^{+}$ trials were shown any more. Since the purpose of the present experiments was to show extinction of inhibition, we wanted to make sure that participants learned the contingencies of the inhibition training cues. We therefore employed a learning criterion at the end of the acquisition stage: Participants had to correctly rate at least $75 \%$ of the last two A, AX, B, and BY trials (i.e., 6 out of a total of 8 trials), respectively.

\section{Method}

Participants. Ninety-one student volunteers were tested individually and needed approximately $15 \mathrm{~min}$ to complete the experiment. Nineteen participants (10 participants in Group Bidirectional and 9 participants in Group Unidirectional) did not reach the learning criterion and were excluded from statistical evaluations. The reported data are based on 72 participants (36 within each group).

Procedure. Instructions and further necessary information were presented on a computer screen. The participants used a computer mouse for responding. The following foods served as cues for the experiment: Bananas, carrots, cherries, grapes, kiwi, mushrooms, pears, peppers, strawberries, and tomatoes. For each participant, these foods were assigned randomly to the different cues.

Participants were told that they should investigate the influence of different foods on the hormone level by examining a group of patients. They were told which food(s) the patients had eaten. On the basis of this information, they had to predict whether they expected the hormone level to increase or remain unchanged (in both groups), or to decrease (in Group Bidirectional). They were informed that feedback after each prediction would allow them to find out how the different foods influence the hormone level. Finally, participants were informed that later in the experiment, they would have to rate to which degree each food influences the hormone level.

Both training stages consisted of six blocks. Each trial type was presented once per block, yielding 48 trials for the acquisition and 36 trials for the extinction stage. The order of presentation was determined randomly for each block. On each trial, participants made their predictions by clicking on a response button. For Group Unidirectional, two buttons were shown labeled "increase" and "no change," respectively. Group Bidirectional was shown an additional third button labeled "decrease." After a participant had made her/his prediction, a feedback window showed the actual outcome of the trial.

Table 1

Design of the Experiments

\begin{tabular}{llll}
\hline \multicolumn{1}{c}{ Type of Trials } & \multicolumn{1}{c}{ Acquisition } & Extinction & \multicolumn{1}{c}{ Test } \\
\hline Inhibition training & $\mathrm{A}^{+}, \mathrm{AX}^{0}$ & $\mathrm{~A}^{+}, \mathrm{X}^{0}$ & $\mathrm{~A}$ ?, X? \\
& $\mathrm{B}^{+}, \mathrm{BY}^{0}$ & $\mathrm{~B}^{+}$ & $\mathrm{B}$ ?, Y? \\
Transfer cue training & $\mathrm{T}^{+}$ & $\mathrm{T}^{+}$ & T?, TX?, TY?, TN? \\
Filler trials & $\mathrm{C}^{0}, \mathrm{DE}^{+}, \mathrm{F}^{0} / \mathrm{F}$ & $\mathrm{C}^{0}, \mathrm{~F}^{0} / \mathrm{F}$ & $\mathrm{C}$ ?, D?, E?, F?, N? \\
\hline
\end{tabular}

Note-The letters depict different cues: ${ }^{+}$means an increase in the hormone level, ${ }^{0}$ means no change of the hormone level, and means a decrease of the hormone level. ? means that participants received no feedback. In both experiments, each participant experienced all stages and all cues with the following exceptions: In Experiment $1, \mathrm{~F}^{0}$ (but no $\mathrm{F}^{-}$) filler trials were used for Group Unidirectional, and $\mathrm{F}^{-}$(but no $\mathrm{F}^{0}$ ) filler trials were used for Group Bidirectional, whereas in Experiment 2, $\mathrm{F}^{-}$(but no $\mathrm{F}^{0}$ ) was used. 
After each learning stage, a test was conducted. Participants used a scale ranging from -10 to +10 to rate to which degree each food influences the hormone level. For both groups, a positive rating indicated that the food increases the hormone level, whereas a neutral rating $(0)$ indicated that the food has no effect. As in studies in which the outcome could vary in only one direction (e.g., Aitken et al., 2000), for Group Unidirectional, the negative pole of the scale was labeled "prevents an increase." For Group Bidirectional, the negative pole was labeled "decreases the level."

In the test, inhibition was assessed in two ways. First, participants had to rate the putative inhibitors $\mathrm{X}_{-}$and $\mathrm{Y}_{-}$on their own, allowing for an assessment of the inhibitory properties of $X_{-}$after acquisition and after extinction. Second, a summation test was conducted in which participants had to rate the new compounds $\left[\mathrm{T}_{+} \mathrm{X}_{-}\right]$and $\left[\mathrm{T}_{+} \mathrm{Y}_{-}\right]$(i.e., $\mathrm{X}_{-}$and $\mathrm{Y}_{-}$as well were combined with the excitatory transfer cue, $\mathrm{T}_{+}$). Furthermore, participants also had to rate additional cues used in the learning stages, and they had to rate a new stimulus, $\mathrm{N}$, that was solely used in the test stage. $\mathrm{N}$ was introduced in order to have a neutral stimulus to which the inhibitory properties of $X_{-}$could also be compared, and to act as a control for the impact of external inhibition. The order of presentation of the different stimuli was determined randomly for each participant. Each test cue and test compound was presented once in each of both tests.

\section{Results and Discussion}

During the acquisition and extinction stages, participants in both groups quickly learned about the experi- mental stimuli so that their predictions mirrored the actual contingencies after approximately the first half of the acquisition stage. Figure 1 shows the mean predictions separately for each cue in each block (top panel, Group Bidirectional; bottom panel, Group Unidirectional). In Group Unidirectional, two alternative predictions had been possible: increase $(=1)$ or constant level $(=0)$. In contrast, in Group Bidirectional, there had been three alternatives: increase $(=1)$, constant level $(=0)$, and decrease $(=-1)$. Analyses of participants' predictions in the extinction stage will be reported later; first, we will focus on the ratings in the two test stages.

Figure 2 shows the mean causal ratings of the single stimuli (F, N, T, X, and Y) and the compounds (TN, TX, and TY) after the acquisition stage (top panel) and after the extinction stage (bottom panel) for both Group Unidirectional (white bars) and Group Bidirectional (black bars). (Note that for the sake of clarity, only the ratings of these eight cues are presented in the figures, although more cues were shown in the test stages.) First, we tested whether $\mathrm{X}$ and $\mathrm{Y}$ were inhibitory after $\mathrm{A}^{+}, \mathrm{AX}^{0}$ training and $\mathrm{B}^{+}, \mathrm{BY}^{0}$ training, by comparing both cues with the neutral stimulus $\mathrm{N}$ in separate ANOVAs. In the first test stage, $\mathrm{X}$ and $\mathrm{Y}$ were rated more negatively than a neutral

\section{Group Bidirectional}

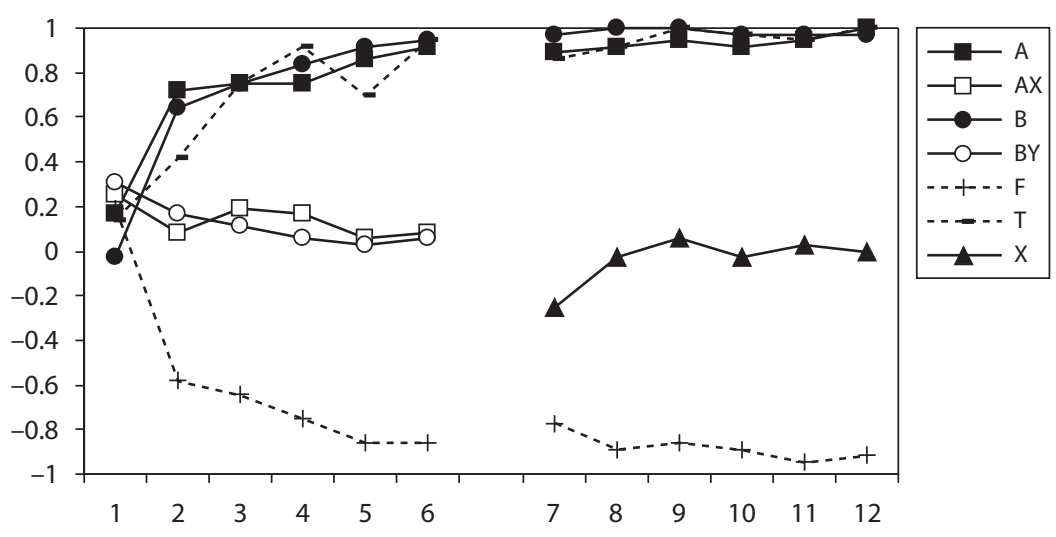

Group Unidirectional

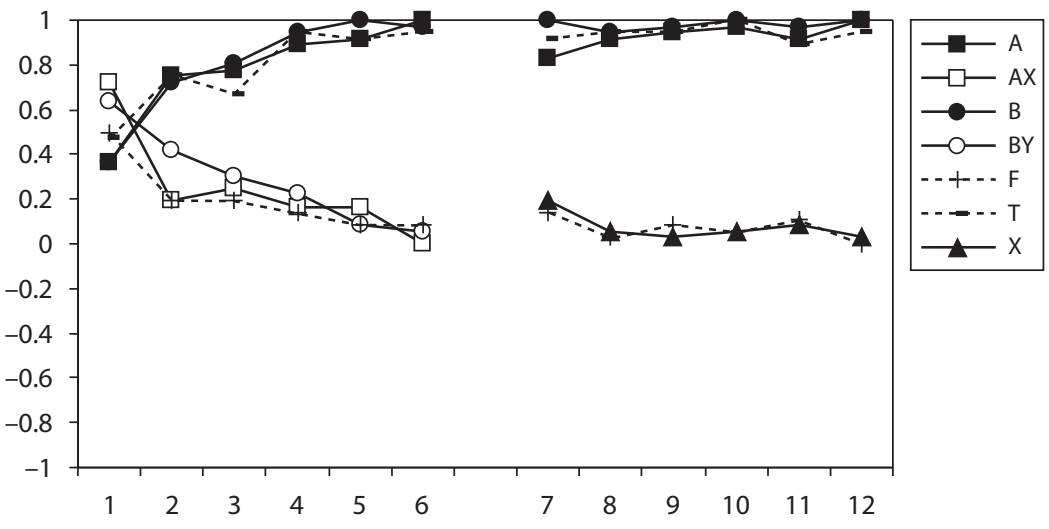

Figure 1. Mean predictions for cues in each block, separate for Group Bidirectional and Group Unidirectional in Experiment 1. The filler cues $C$ and DE are not shown. 
stimulus $\mathrm{N}$ in both groups. We found a significant main effect of stimulus for $\mathrm{X}[F(1,70)=48.58, p<.001]$ and $\mathrm{Y}[F(1,70)=40.06, p<.001]$ as well. Furthermore, in the summation tests, $\operatorname{TX}[F(1,70)=32.27, p<.001]$ and TY $[F(1,70)=53.59, p<.001]$ were rated more negative than $\mathrm{TN}$, excluding external inhibition as an alternative explanation of conditioned inhibition. In neither analysis did we find a significant stimulus $\times$ group interaction, reflecting the fact that there was no evidence of a difference between the groups. The ANOVA contrasting $\mathrm{X}$ and $\mathrm{N}$ across the two groups, however, revealed a significant main effect of group $[F(1,70)=4.43, p<.05]$, indicating that both cues were rated more positively in Group Bidirectional than in Group Unidirectional. No such effect of group was found in the ANOVA contrasting $\mathrm{Y}$ and $\mathrm{N}$, or in any other analysis (all $F \mathrm{~s}<1)$.

The inhibitors $\mathrm{X}$ and $\mathrm{Y}$ had received identical training in the acquisition stage and thus should be rated similarly in the first test. Nevertheless, the bars in the top panel of Figure 2 suggest that $\mathrm{X}$ was rated less negatively than $\mathrm{Y}$ in Group Bidirectional, indicating that the putative inhibitor X might not have been learned sufficiently. In addition, the bars suggest that $\mathrm{X}$ was rated more negatively in Group Unidirectional as compared with Group Bidirectional. Because we expect extinction of $\mathrm{X}$ in Group Bidirectional, but not in Group Unidirectional, this might be a problem (a kind of floor effect for extinction of inhibition in case of $\mathrm{X}$ ). Thus, in additional ANOVAs, we compared the ratings of $\mathrm{X}$ and $\mathrm{Y}$, as well as the ratings of TX and TY. Regarding $\mathrm{X}$ and $\mathrm{Y}$, the main effect of stimulus was not significant $(F<1)$, but the stimulus $\times$ group interaction was $[F(1,70)=5.89, p<.05]$. Thus, overall, there was no evidence of a difference between $\mathrm{X}$ and $\mathrm{Y}$, but the difference in magnitude differed between the groups. Further analyses revealed that the main effect of stimulus within each group failed to reach significance in Group Bidirectional $[F(1,35)=2.21, p>.10]$, but was significant in Group Unidirectional $[F(1,35)=4.13, p<.05]$. The ratings of TX and TY showed no significant differences at all (all $F$ s $<1$ ). In neither analysis did we find a significant main effect of group.

Since differences in the ratings of the inhibitors $\mathrm{X}$ and $\mathrm{Y}$ before the extinction stage might be a problem for interpreting the data, we calculated additional analyses with a subset of participants. We selected participants whose ratings of $\mathrm{X}$ and $\mathrm{Y}$ at the end of the acquisition stage did not differ as much as in the whole sample (i.e., the withinsubjects difference was less than 10 rating steps). After the exclusion of 12 (out of 72) participants, we found no significant difference between either X and Y (as can be expected due to the selection) or TX and TY at the end of acquisition. With the data of the selected sample, we then calculated the same analyses of the ratings after the extinction stage, as reported below. In the selected sam-

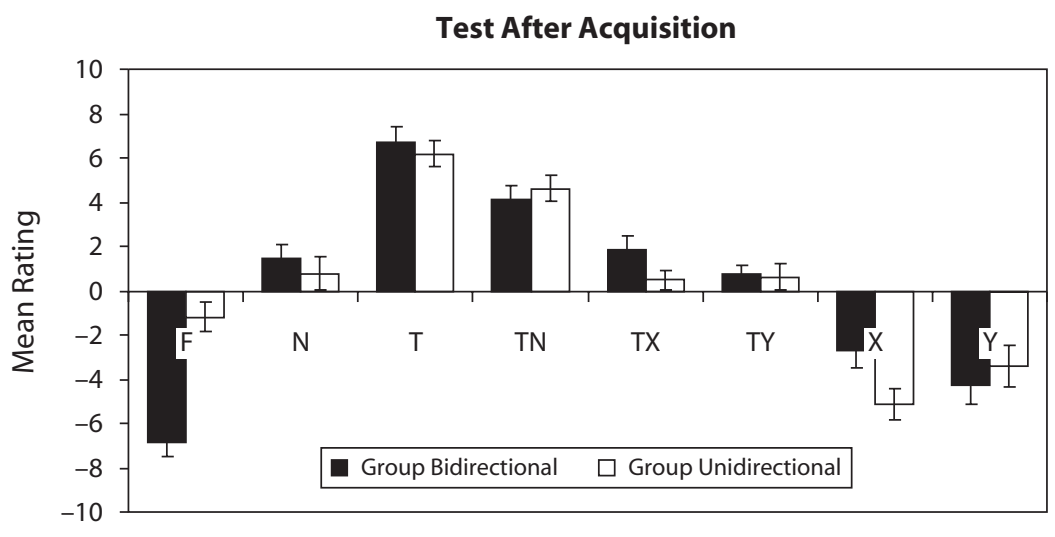

Test After Extinction

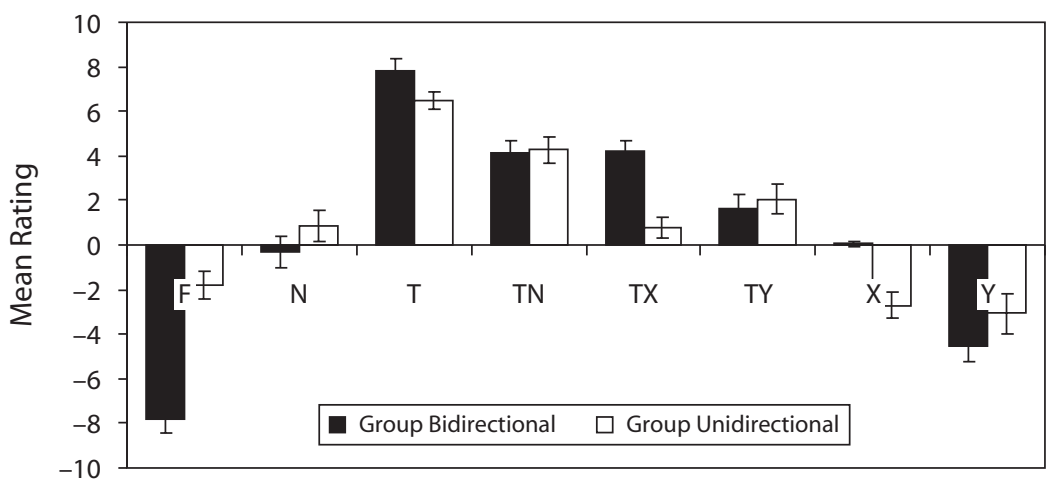

Figure 2. Mean causal ratings after the acquisition stage and after the extinction stage in Experiment 1. 
ple, we found exactly the same pattern of results as in the unselected sample that will be reported presently. Thus, postextinction differences between groups (that we expect) cannot be attributed to preextinction differences in the ratings of $\mathrm{X}$ and $\mathrm{Y}$.

In the extinction stage, the inhibitor $\mathrm{X}_{-}$was presented with the neutral outcome "level remains unchanged" $\left(\mathrm{X}_{-}^{0}\right)$. Thus, in the following test, we expected $\mathrm{X}_{-}$to be extinguished in Group Bidirectional but not in Group Unidirectional. The bottom panel of Figure 2 shows the ratings in the second test stage (after extinction). Successful extinction can be assumed, if $\mathrm{X}$ is rated more positively than $\mathrm{Y}$ in the direct comparison, and if TX is rated more positively than TY in the summation test. We calculated the relevant ANOVAs and found a significant main effect of stimulus $[F(1,70)=17.28, p<.001]$ and a significant stimulus $\times$ group interaction for the comparison of $X$ and $\mathrm{Y}[F(1,70)=12.57, p<.001]$. The main effect of group was not significant $(F<1)$. Furthermore, the comparison of TX and TY revealed a significant main effect of group $[F(1,70)=7.01, p<.01]$ and a significant stimulus $\times$ group interaction $[F(1,70)=13.02, p<.001]$, but no significant main effect of stimulus $[F(1,70)=1.45, p>.20]$. In Group Bidirectional, the main effects of stimulus were significant for the comparison of $\mathrm{X}$ and $\mathrm{Y}[F(1,35)=$ $38.18, p<.001]$ and of TX and TY $[F(1,35)=12.38$, $p<.001]$. In Group Unidirectional, however, the main effects of stimulus were not significant, for either the comparison of $\mathrm{X}$ and $\mathrm{Y}(F<1)$ or the comparison of TX and TY $[F(1,35)=2.72, p>.10]$. Thus, we concluded that successful extinction occurred in Group Bidirectional, but not in Group Unidirectional.

The comparison of $\mathrm{X}$ and $\mathrm{Y}$ will demonstrate extinction of $X$ only if $Y$ can still be shown to be inhibitory. Thus, we additionally compared ratings of $Y$ and $N$ and ratings of $T Y$ and TN in both groups and found a significant main effect of stimulus $[F(1,70)=40.88, p<.001$, and $F(1,70)=$ $18.82, p<.001]$, but no significant group $\times$ stimulus interaction and no significant main effect of group [for comparison of $\mathrm{Y}$ and $\mathrm{N}$, we found no significant main effect of group, $F(1,70)=2.16, p>.10$; all other $F$ s $<1$ ]. These results show that $Y$ was still inhibitory after the extinction of $X$ in both groups. In contrast, a comparison of $\mathrm{X}$ and $\mathrm{N}$ and a comparison of TX and TN after extinction showed a significant main effect of stimulus $[F(1,70)=$ $7.07, p<.01$, and $F(1,70)=14.04, p<.001]$, and a significant group $\times$ stimulus interaction $[F(1,70)=10.59$, $p<.01$, and $F(1,70)=15.44, p<.001]$. The main effect of group was significant for the comparison of TX and $\mathrm{TN}[F(1,35)=7.89, p<.01]$ but was not significant for the comparison of $\mathrm{X}$ and $\mathrm{N}[F(1,35)=2.15, p>.10]$. In Group Bidirectional, ratings of N and X and of TN and TX did not differ $(F \mathrm{~s}<1)$, whereas a significant difference was evident in Group Unidirectional $[F(1,35)=13.70$, $p<.001$, and $F(1,35)=30.32, p<.001$, indicating that in the latter group, $\mathrm{X}$ was still inhibitory.

Successful extinction in Group Bidirectional was further supported by analyses of the course of predictions for $\mathrm{X}$ across blocks during extinction (see Figure 1). Because of the difference in the scale (0 to 1 in Group Unidirec- tional and -1 to 1 in Group Bidirectional), extinction in the course of predictions cannot be interpreted in terms of group differences. Thus, analyses were conducted separately for each group. Paired $t$ tests for the predictions in the first and third extinction blocks (Block 7 and Block 9) showed that in Group Bidirectional, mean predictions for $\mathrm{X}$ significantly increased from a negative value in Block 7 to a value near 0 in Block $9[t(35)=-2.33, p<.05]$, whereas in Group Unidirectional, these predictions significantly decreased from an initially positive value in Block 7 to a value near 0 in Block $9[t(35)=2.65, p<$ $.05]$. Thus, in Group Bidirectional, the course of predictions equaled extinction of an inhibitor, whereas in Group Unidirectional, $\mathrm{X}$ became more negative.

Overall, the results suggest that successful extinction only took place in Group Bidirectional, in which the outcome varied on a bidirectional continuum. Thus, in Experiment 1, we were able to replicate Melchers et al.'s (2006) basic observation. Furthermore, we successfully tested the potential inhibitor directly against a second inhibitor within subjects, and demonstrated that extinction of inhibition is cue specific. And, last but not least, we could observe extinction "online" by comparing participants" predictions across blocks within the extinction stage.

A potential problem with Experiment 1 was that the values of $\mathrm{X}$ and $\mathrm{Y}$ differed at the end of acquisition - $\mathrm{a}$ fact that might have complicated the interpretation of the data after extinction (although we showed that the pattern of results in the whole sample was identical to the pattern of results in a selected sample without postacquisition differences; see above). We decided to slightly alter conditions in the subsequent experiment in three ways. First, we lengthened the acquisition stage by two more blocks to enhance the chance for asymptotic learning at the end of the acquisition stage. Second, in order to reduce random variations in the ratings, we introduced a replication in the tests: Each stimulus was rated twice in each test. Third, we changed the rating scale from 21 steps $(-10$ to +10$)$ into 11 steps $(-5$ to +5$)$.

\section{EXPERIMENT 2}

Experiment 2 addressed a potentially problematic issue related to procedural differences in the learning stages between the bidirectional groups and typical causal learning studies: A doubt about the validity of the conclusions drawn so far could be that the results are determined not by the nature of the outcome continua, but by other particulars of the procedure. During the extinction phase, only Group Bidirectional but not Group Unidirectional is taught a response to stimulus $\mathrm{X}$ that is inconsistent with its presumed inhibitory learning. If stimulus $\mathrm{X}$ acquires negative $V$ in both groups, this negative $V$ would lead to the expectation that if the stimulus was presented on its own, it should cause a decrease in hormone levels. During the extinction phase, participants in Group Bidirectional have the "decrease" button as a response option, but participants in Group Unidirectional do not. Thus, only participants in Group Bidirectional must learn to select the "no change" button instead of the "decrease" button 
during the extinction phase, resulting in an extinction effect. Hence, this effect could occur as a result of a kind of counterconditioning manipulation applied to Group Bidirectional (assuming that the different outcomes are distinct USs). To deal with this objection, in Experiment 2, we again examined extinction of inhibition through both the pairing of the inhibitor with a neutral outcome and the use of a bidirectional outcome continuum, but we modified the learning situation to avoid the problems just noted. Specifically, participants only had to inspect the cue-outcome relationships without corrective feedback to shape their responses - a procedure that is not uncommon in causal learning (see, e.g., Pineño, Denniston, Beckers, Matute, \& Miller, 2005; Vandorpe, de Houwer, \& Beckers, 2007). Moreover, a procedure in which participants only observe might be thought to increase comparability with various other associative learning experiments, such as human skin conductance learning. Hence, in Experiment 2, participants solely had to observe cues and outcomes without making predictions. In the test stages, cues were rated in the same manner as in Experiment 1 (except for the slightly modified rating scale; see above). Otherwise, the same medical prediction task as in Experiment 1 was used.

To ensure that participants paid enough attention to the stimuli and outcomes in a setting in which information is solely observed, we combined two methods described in former studies (Pineño et al., 2005; Vandorpe et al., 2007). Cues and outcomes were presented successively with a short delay, and a further delay ensured that the presented information remained on the screen for at least 2 sec. Furthermore, after each learning trial, a posttrial test was conducted in which participants had to choose the presented cues out of all stimuli used in Experiment 2.

\section{Method}

Participants. Thirty-six student volunteers were tested individually and needed approximately $20 \mathrm{~min}$ to complete the experiment.

Procedure. Instructions were similar to those used in Experiment 1 . The following foods served as cues for the experiment: bananas, carrots, kiwi, lemons, mushrooms, pears, peppers, potatoes, strawberries, and tomatoes. The foods were assigned randomly to the different cues, individually for each participant.

The acquisition stage consisted of eight blocks, and the extinction stage consisted of six blocks. Each trial type was presented once per block, yielding 64 trials for the acquisition and 36 trials for the extinction stage. The order of presentation was determined randomly for each block. Each trial started with the presentation of a cue (picture[s] and name[s] of the food[s]) in the center of the screen. After approximately $3,000 \mathrm{msec}$, the current value of the outcome was presented beneath the cue; the hormone level could increase, remain unchanged, or decrease. After a further delay of $2,000 \mathrm{msec}$, a button was enabled on which participants could click to end the trial. Subsequently, a posttrial test was conducted in which all stimuli were presented on the screen simultaneously. Participants were asked which of the foods the patient had consumed on the current trial. They made their choices by clicking the corresponding pictures. After completion, the next trial started.

After each learning stage, a test was conducted in which participants had to rate to which degree each food influences the hormone level, using a rating scale ranging from -5 to +5 . Each test cue and test compound was presented two times in each test. Further details of the test stages are as described in the Method section of Experiment 1.

\section{Results and Discussion}

Figure 3 shows the mean causal ratings of the stimuli $\mathrm{F}, \mathrm{N}, \mathrm{T}, \mathrm{X}$, and $\mathrm{Y}$, as well as the mean ratings of the compounds TN, TX, and TY after the acquisition stage (top panel) and the extinction stage (bottom panel). We tested whether $\mathrm{X}$ and $\mathrm{Y}$ were inhibitory after $\mathrm{A}^{+}, \mathrm{AX}^{0}$ training and $\mathrm{B}^{+}, \mathrm{BY}^{0}$ training in the acquisition stage by comparing both cues with the neutral stimulus $N$ in separate ANOVAs. Stimuli $\mathrm{X}$ and $\mathrm{Y}$ both were rated significantly more negative than was $\mathrm{N}$. We found a significant main effect of stimulus for the comparison of $\mathrm{X}$ and $\mathrm{N}[F(1,35)=43.98$, $p<.001]$, and for the comparison of $\mathrm{Y}$ and $\mathrm{N}[F(1,35)=$ $39.16, p<.001]$. Furthermore, TX and TY were rated significantly more negative than $\mathrm{TN}$ in the summation test, as indicated by significant main effects of stimulus [TX vs. $\mathrm{TN}, F(1,35)=341.32, p<.001$; TY vs. TN, $F(1,35)=$ 369.63, $p<.001]$. In additional ANOVAs, we compared the ratings of $\mathrm{X}$ and $\mathrm{Y}$ and the ratings of TX and TY after the acquisition stage. Regarding $X$ and $Y$, the analysis suggested that the ratings did not differ (main effect of stimulus: $F<1$ ). The ratings of TX and TY did not differ either (main effect of stimulus: $F<1$ ). Thus, both inhibitors $\mathrm{X}$ and $\mathrm{Y}$ were learned equally.

In the extinction stage, the inhibitor $\mathrm{X}_{-}$was presented with a neutral outcome $\left(\mathrm{X}_{-}^{0}\right)$. In the following test, we therefore expected $\mathrm{X}_{-}$to be extinguished. The bottom panel of Figure 3 shows the ratings in the test stage after extinction. We found significant main effects of stimulus for the comparison of X and $\mathrm{Y}[F(1,35)=30.74, p<$ $.001]$ and for the comparison of TX and TY $[F(1,35)=$ $17.97, p<.001$ ], indicating that successful extinction had taken place. A comparison of TY and TN, as well as a comparison of $\mathrm{Y}$ and $\mathrm{N}$ after the extinction stage, showed that $Y$ was still inhibitory $[F(1,35)=95.56, p<.001$, and $F(1,35)=12.61, p<.001]$. Thus, the results strengthen the view that in the previous experiments, the changes in the ratings of $\mathrm{X}$ had been due to extinction as well.

In comparison with Experiment 1, however, in Experiment 2 , the ratings of the excitor $T_{+}$were less positive, and the ratings of the new compound TN were much more positive. Paired $t$ tests showed that TN was rated significantly higher than $\mathrm{T}$ after acquisition $[t(35)=-5.45, p<.001]$ and after extinction $[t(35)=-3.80, p<.001]$. Whereas Experiment 1 showed a decrease from T to TN, in Experiment 2, we observed a significant increase from $\mathrm{T}$ to $\mathrm{TN}$. The latter finding is interesting, since external inhibition, which can be supposed to be the reason for lower ratings of $\mathrm{TN}$ in Experiment 1, seems to play no role in Experiment 2. The results might indicate that the changes in procedure had effects on the perception of additivity of the cues. In line with this suggestion, we found that after acquisition in Experiment 2, the ratings of $\mathrm{X}$ and $\mathrm{Y}$ were more negative than in Experiment 1 (according to the Rescorla-Wagner theory, the inhibitory $V$ of these cues also results from an additivity process). We are not aware of studies in which similar training arrangements (training with and without predictions) have been compared systematically. This potential difference might be worth further research, since the two training arrangements are often treated as equivalent in human causal learning studies. 


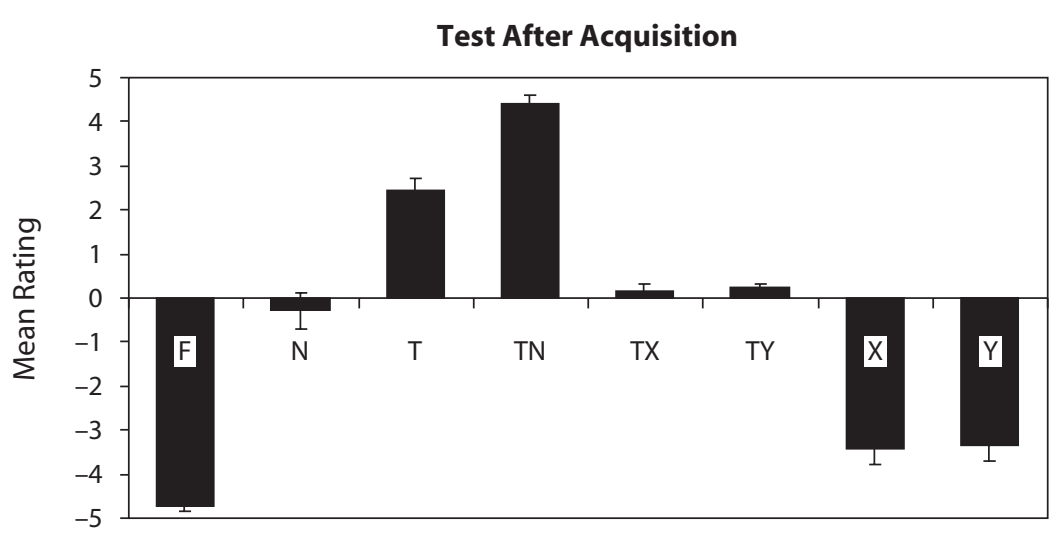

Test After Extinction

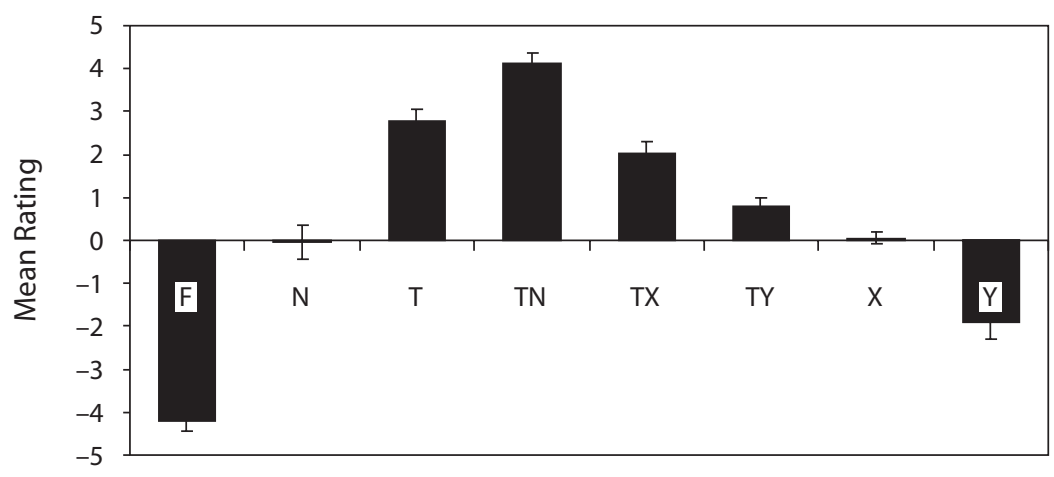

Figure 3. Mean causal ratings after the acquisition stage and after the extinction stage in Experiment 2.

\section{GENERAL DISCUSSION}

In two experiments, we investigated extinction of inhibition through the pairing of the inhibitor with a neutral outcome. In Experiment 1, we replicated and extended findings of Melchers et al. (2006). Successful extinction was demonstrated, if and only if a bidirectional outcome continuum was used that empirically reflected the symmetry of excitation and inhibition assumed in the RescorlaWagner theory. With a traditional unidirectional continuum, however, extinction failed, as had been repeatedly observed before. We established a within-subjects control for extinction by comparing the (presumably) extinguished inhibitor with a second inhibitor that had not been presented with a neutral outcome in the extinction stage. In this way, we were able to demonstrate cue specifity of extinction of inhibition. Comparing an extinguished and a nonextinguished cue at the time of testing allowed us to demonstrate the effectiveness of the extinction process. Experiment 2 replicated the basic results from Experiment 1 and excluded potential procedural problems by altering the training conditions from prediction to mere observation. The observed patterns of the measures of inhibition after acquisition and extinction were the same as in Experiment 1.

An inspection of the ratings in both experiments (Figures 1 and 3) reveals that the second inhibitor, Y, which had not been presented with the neutral outcome in the ex- tinction stage, tended to be rated less negatively after the extinction stage than before. We suppose that this effect might have been due to forgetting, because $Y$ was presented during the acquisition stage but not during the extinction stage. Nevertheless, in tests with bidirectional scales, we found significant stimulus effects, indicating that the extinguished inhibitor $\mathrm{X}$ was rated less negative than Y. If the assumption of forgetting should be correct, then the present within-subjects tests underestimate the extinction effect. This issue, however, could be solved in subsequent studies by adding $\mathrm{BY}^{0}$ trials to the extinction stage (provided that BY is already trained asymptotically).

As was already mentioned, the results of the bidirectional groups are in support of the Rescorla-Wagner assumption of inhibition being the symmetrical counterpart of excitation. In its present form, however, the RescorlaWagner theory cannot deal with the results in the unidirectional group (which is the standard case in associative learning). Thus, the Rescorla-Wagner theory cannot explain the discrepancy between extinction effects with unidirectional and bidirectional continua. On the other hand, theories that challenged this conception of inhibition also conflict with the present data: They either predict extinction of inhibition or the absence of an extinction effect; they cannot predict the observed pattern of results. Threshold accounts of inhibition (see, e.g., Konorski, 1948; Rescorla, 1979), which propose that conditioned inhibition increases a threshold for activation of the US, wrongly 
assume that inhibition does not extinguish with unpaired presentations of the inhibitor. Since X solely raises the threshold for US activation, it has no effect on its own; hence, inhibition may not extinguish. The same is true for the view of conditioned inhibition that assumes that associations between the CS and no-US centers are established and subtracted from excitatory associations between the CS and US centers (Konorski, 1967). In both cases, the extension of the outcome continuum by adding a negative outcome does not change the associations of the inhibitor. This is because in acquisition, the inhibitor is established through pairings with the neutral outcome (in compound with an excitatory cue), and in extinction, it is again paired with the neutral outcome. Hence, the inhibitor (X) is always paired with a neutral outcome (and may be affected by the positive outcome through compound presentations with an excitatory cue (AX). Since the inhibitor is never paired with the negative outcome, the mere existence of a negative outcome or its occurrence during the experiment $\left(\mathrm{F}^{-}\right.$trials) has no effect, regardless of being coded as negative value or as a distinct second US.

A further approach - the comparator hypothesis (Miller \& Matzel, 1988), and its more recent mathematical implementation, SOCR (Stout \& Miller, 2007) — assumes that no inhibitory associations exist at all: Inhibitory behavior is the result of weaker associations between a CS and the US than between a comparator stimulus and the US (given a strong $\mathrm{CS}$-comparator stimulus association). According to this approach, during acquisition, $\mathrm{A}$ is the comparator for $\mathrm{X}$. During extinction training, however, the $\mathrm{X}^{0}$ trials strengthen the $\mathrm{X}$-context association and weaken the direct $\mathrm{X}-\mathrm{A}$ association, causing the context to become a more potent comparator stimulus for $\mathrm{X}$ than for $\mathrm{Y}$ (personal communication with Ralph Miller, May 8, 2008). Hence, this view correctly predicts the extinction of inhibition in the bidirectional groups. According to SOCR, comparisons only occur between cues that are connected by within-compound associations. The mere presence or absence of a negative outcome ( $\mathrm{F}^{-}$trials in the bidirectional groups, but not in the unidirectional group) should have no consequence (personal communication with Ralph Miller, May 9, 2008). Therefore, SOCR does not easily account for the unidirectional case.

The contrast-effects literature (for an overview, see Mackintosh, 1974) offers a mechanism to explain differences in extinction between the groups on the basis of the presence or absence of a negative outcome. In this view, in the unidirectional group $\mathrm{F}^{0}$ and $\mathrm{X}^{0}$, trials in the extinction stage should result in no change of ratings of $\mathrm{X}$. In contrast, due to presentations of $\mathrm{X}^{0}$ with spatiotemporal proximity to a negative $\mathrm{F}^{-}$in bidirectional groups, $\mathrm{X}$ should be rated more positive at the end of the extinction stage, since there is an obvious contrast between both cues. In support of this view, we found a (statistically nonsignificant) trend that showed that the neutral cue (C) was rated more excitatory in the bidirectional group than in the unidirectional group, at the end of both the acquisition stage and the extinction stage. To test this approach, studies with and without an explicitly negative cue should be conducted.
A more recent position assuming higher order reasoning processes in causal learning (see, e.g., De Houwer, Beckers, \& Vandorpe, 2005; Lovibond, 2003) is also in accord with the present results. In this view, people infer by controlled and effortful processing that a cue is a valid cause of an outcome. In the unidirectional group of Experiment 1, participants could have inferred that the putative inhibitors $\mathrm{X}$ and $\mathrm{Y}$ prevent an increase of the hormone level after the acquisition stage. Presentations of $\mathrm{X}$ with a neutral outcome in the extinction stage should not modify this inference, since X might still prevent an increase, if it were presented in compound with an excitor. In contrast, in the bidirectional group, people could infer that $\mathrm{X}$ and $\mathrm{Y}$ reduce the hormone level. Thus, after presentations of $\mathrm{X}$ with a neutral outcome in the extinction stage, they should infer that there was a change because $X$, instead of reducing the level, apparently now has no effect on the hormone level. This observation then results in extinction of inhibition. This inferential account is not limited to human causal learning; evidence also comes from human skin conductance conditioning experiments (Mitchell \& Lovibond, 2002). Furthermore, recently Beckers, Miller, De Houwer, and Urushihara (2006) found evidence for the inferential account in Pavlovian conditioning of rats as well.

Together with those findings, our results highlight the sensitivity of learners to aspects such as the nature of the outcome and the necessity to take this into account. Yet, this does not necessarily refute associative theories as possible accounts of how humans learn about relationships between cues and their potential outcomes (see, e.g., Lober \& Shanks, 2000; Melchers et al., 2004). Further research might take contrast effects into account, which could be a possible explanation for effects of different outcome properties. Moreover, it would be interesting to show extinction of inhibition through nonreinforced presentations of the inhibitor in animal learning. Since there are few (if any) USs that might take on positive as well as negative values, different levels of the US (i.e., the absence of the US, a small US intensity, and a large US intensity) might be used (see, e.g., Cotton, Goodall, \& Mackintosh, 1982).

\section{AUTHOR NOTE}

This research was supported by Grant LA 564/18-1 from the Deutsche Forschungsgemeinschaft to H.L. We thank Ralph Miller for substantial suggestions regarding SOCR and for drawing our attention to the contrast effects account. Furthermore, we are deeply indebted to Klaus Melchers, who, being overmodest, decided not to become a coauthor of the present article. We also thank Jana Anding for her help in collecting the data and with the preparation of the manuscript. Correspondence concerning this article should be addressed to A. Lotz, Philipps-Universität Marburg, Department of Psychology, Gutenbergstr., 18, D-35032 Marburg, Germany (e-mail: lotza@staff.uni-marburg.de).

\section{REFERENCES}

Aitken, M. R. F., Larkin, M. J. W., \& Dickinson, A. (2000). Superlearning of causal judgments. Quarterly Journal of Experimental Psychology, 53B, 59-81.

Beckers, T., Miller, R. R., De Houwer, J., \& Urushihara, K. (2006). Reasoning rats: Forward blocking in Pavlovian animal conditioning is sensitive to constraints of causal inference. Journal of Experimental Psychology: General, 135, 92-102. 
Chapman, G. B., \& Robbins, S. J. (1990). Cue interaction in human contingency judgment. Memory \& Cognition, 18, 537-545.

Cotton, M. M., Goodall, G., \& Mackintosh, N. J. (1982). Inhibitory conditioning resulting from a reduction in the magnitude of reinforcement. Quarterly Journal of Experimental Psychology, 34B, 163-180.

De Houwer, J., Beckers, T., \& Vandorpe, S. (2005). Evidence for the role of higher order reasoning processes in cue competition and other learning phenomena. Learning \& Behavior, 33, 239-249.

Karazinow, D. M., \& BoAKES, R. A. (2004). Learning about cues that prevent an outcome: Conditioned inhibition and differential inhibition in human predictive learning. Quarterly Journal of Experimental Psychology, 57B, 153-178.

KonORSKI, J. (1948). Conditioned reflexes and neuron organization. Cambridge: Cambridge University Press.

KONORSKI, J. (1967). Integrative activity of the brain: An interdisciplinary approach. Chicago: University of Chicago Press.

Lober, K., \& Shanks, D. R. (2000). Is causal induction based on causal power? Critique of Cheng (1997). Psychological Review, 107, 195-212.

Lovibond, P. F. (2003). Causal beliefs and conditioned responses: Retrospective revaluation induced by experience and by instruction. Journal of Experimental Psychology: Learning, Memory, \& Cognition, 24, 1331-1352.

Lovibond, P. F., Been, S.-L., Mitchell, C. J., Bouton, M. E., \& FroHARDT, R. (2003). Forward and backward blocking of causal judgments is enhanced by additivity of effect magnitude. Memory \& Cognition, 31, 133-142.

Mackintosh, N. J. (1974). The psychology of animal learning. London: Academic Press.

Melchers, K. G., Lachnit, H., \& Shanks, D. R. (2004). Withincompound associations in retrospective revaluation and in direct learning: A challenge for comparator theory. Quarterly Journal of Experimental Psychology, 57B, 25-53.

Melchers, K. G., WolfF, S., \& Lachnit, H. (2006). Extinction of conditioned inhibition through nonreinforced presentation of the inhibitor. Psychonomic Bulletin \& Review, 13, 662-667.

Miller, R. R., \& Matzel, L. D. (1988). The comparator hypothesis: A response rule for the expression of associations. In G. H. Bower (Ed.), The psychology of learning and motivation (Vol. 22, pp. 51-92). San Diego: Academic Press.

Miller, R. R., \& Schachtman, T. R. (1985). Conditioning context as an associative baseline: Implications for response generation and the nature of conditioned inhibition. In R. R. Miller \& N. E. Spear (Eds.), Information processing in animals: Conditioned inhibition (pp. 51-88). Hillsdale, NJ: Erlbaum

Mitchell, C. J., \& Lovibond, P. F. (2002). Backward and forward blocking in human electrodermal conditioning: Blocking requires an assumption of outcome additivity. Quarterly Journal of Experimental Psychology, 55B, 311-329.

Pearce, J. M. (1987). A model for stimulus generalization in Pavlovian conditioning. Psychological Review, 94, 61-73.

Pineño, O., Denniston, J. C., Beckers, T., Matute, H., \& Miller, R. R. (2005). Contrasting predictive and causal values of predictors and of causes. Learning \& Behavior, 33, 184-196.

Rescorla, R. A. (1979). Conditioned inhibition and extinction. In A. Dickinson \& R. A. Boakes (Eds.), Mechanisms of learning and motivation: A memorial volume to Jerzy Konorski (pp. 83-110). Hillsdale, NJ: Erlbaum.

Rescorla, R. A., \& Wagner, A. R. (1972). A theory of Pavlovian conditioning: Variations in the effectiveness of reinforcement and nonreinforcement. In A. H. Black \& W. F. Prokasy (Eds.), Classical conditioning II: Current theory and research (pp. 64-99). New York: Appleton-Century-Crofts.

Stout, S. C., \& Miller, R. R. (2007). Sometimes-competing retrieval (SOCR): A formalization of the comparator hypothesis. Psychological Review, 114, 759-783.

Vandorpe, S., De Houwer, J., \& Beckers, T. (2007). The role of memory for compounds in cue competition. Learning \& Motivation, 38, 195-207.

Williams, D. A., Overmier, J. B., \& LoLordo, V. M. (1992). A reevaluation of Rescorla's early dictums about Pavlovian conditioned inhibition. Psychological Bulletin, 111, 275-290.

Zimmer-Hart, C. L., \& Rescorla, R. A. (1974). Extinction of Pavlovian conditioned inhibition. Journal of Comparative \& Physiological Psychology, 86, 837-845.

\section{NOTE}

1. We use the icons,+ 0 , and - as subscripts to refer to the assumed associative status of a CS: A CS that is assumed to be excitatory is referred to as $\mathrm{CS}_{+}$; a $\mathrm{CS}$ that is assumed to have an associative strength of 0 is referred to as $\mathrm{CS}_{0}$; and a putative inhibitor CS is referred to as $\mathrm{CS}_{-}$. Furthermore, we use these three icons as superscripts to specify the experimental procedure - that is, the type of outcome. A CS paired with a positive outcome is referred to as $\mathrm{CS}^{+}$; a CS paired with a neutral outcome is referred to as $\mathrm{CS}^{0}$, and a $\mathrm{CS}$ paired with a negative outcome is referred to as CS - For example, the extinction of an excitatory CS by presenting it without US is referred to as $\mathrm{CS}_{+}^{0}$, and the extinction of an inhibitory $\mathrm{CS}$ by presenting it without US is referred to as $\mathrm{CS}_{-}^{0}$.

(Manuscript received May 19, 2008; revision accepted for publication August 15, 2008.) 